\title{
LA COMUNICACIÓN NO VERBAL Y EL ESTUDIO DE SU INCIDENCIA EN FENÓMENOS DISCURSIVOS COMO LA IRONÍA ${ }^{1}$
}

\author{
Ana $M^{a}$ Cestero Mancera \\ Universidad de Alcalá \\ anam.cestero@uah.es
}

\begin{abstract}
Resumen
En este artículo se presenta la comunicación no verbal y se detallan los sistemas que la integran, prestando especial atención a los signos de los sistemas más relacionados con la comunicación oral humana, por su incidencia determinante en la construcción e interpretación de fenómenos pragmático-discursivos como la ironia verbal. Se ofrecen, además, algunas ideas y propuestas básicas para abordar su estudio de manera sistemática.
\end{abstract}

PALABRAS CLAVE: comunicación no verbal, paralenguaje, quinésica, discurso, ironía verbal.

\begin{abstract}
This article presents non-verbal communication as well as an exhaustive vision of the systems cmbodied in it. The signs of those systems, further related to oral communication, are specially studied due to its determining effect on the construction and interpretation of pragmatic-discursive facts such as verbal irony. Furthermore some ideas and basic motions are proposed to undertake a systematic study on this matter
\end{abstract}

KEY WORDS: Nonverbal Communication, Paralenguage, Kinesics, Discourse, Verbal Irony.

\section{La comunicación no verbal}

La expresión comunicación no verbal alude a todos los signos y sistemas de signos no lingüísticos que comunican o se utilizan para comunicar. Como puede apreciarse por su definición, se trata de un concepto extraordinariamente amplio, que incluye los hábitos y las costumbres culturales y los denominados sistemas de comunicación no verbal. La amplitud y complejidad del tema explican el lento desarrollo y la interdisciplinariedad que caracterizan su estudio.

El conocimiento que hoy en día tenemos sobre comunicación no verbal es relativamente escaso y muy fragmentario. Tanto es así que, a comienzos del siglo XXI, aún no contamos con una base teórica sólida que nos permita describir y explicar con propiedad y detalle qué es la comunicación no verbal, qué sistemas la integran, cuáles son sus signos y cómo funcionan, debido, fundamentalmente, a la dificultad metodológica que entraña su investigación y a la poca y heterogénea tradición que tiene su estudio (Serrano, 1981; Poyatos, 1994b).

1 Este artículo fue pronunciado como conferencia, con el título "La Comunicación no Verbal y su estudio", en las VIII Jornadas de Estudios de Lingüistica. Anúlisis del Discurso, celebradas en la Universidad de Alicante, los días 14,15 y 16 de marzo de 2006 . 
Se pueden encontrar alusiones a los signos no verbales y descripciones de los mismos dispersas por las obras de clásicos latinos y griegos o en tratados filosóficos y estudios de medicina, antropológicos, sociales, históricos e, incluso, lingüísticos de siglos pasados; pero se considera que la primera obra que versa en exclusividad sobre comunicación no verbal es The Expression of the Emotions in Man and Animals de Ch. Darwing, publicada en 1872. De ella parte la investigación sobre comunicación no verbal posterior, muy escasa en la primera mitad del siglo XX, aunque cuenta con la obra de Sapir (1949), en la que se destaca la importancia de los signos no verbales en la comunicación humana, y bastante fructífera en la segunda mitad, en la que nace la Comunicación no Verbal como disciplina, a la par que las ramas de la Lingüística que trabajan con el habla y que otras corrientes sociológicas, antropológicas, etc. que se centran en la comunicación humana. A finales de los años cincuenta, antropólogos como R. Birdwhistell y E. T. Hall presentan sus estudios sobre quinésica y proxémica y cronémica, respectivamente, $\mathrm{y}$, por las mismas fechas, psicólogos y psiquiatras publican trabajos en los que se analiza el comportamiento no verbal de pacientes, dando origen a la corriente de investigación conocida como Comunicación no Verbal. En las décadas siguientes se produce su consolidación, nuevamente a partir de los trabajos de especialistas procedentes de distintas ciencias: la antropología, la psicología, la lingüística y la sociología fundamentalmente.

La investigación en Comunicación no Verbal, no obstante, no se lleva a cabo, exclusivamente, desde la disciplina que lleva su nombre, y que tiene como objeto de estudio principal los signos y actos de comunicación producidos a partir de signos que pertenecen a los denominados sistemas de comunicación no verbal, sino que se hace también, y de forma muy precisa, desde la corriente de corte sociológico denominada Análisis de la Conversación, cuyo objetivo es descubrir y documentar las organizaciones sistemáticas del habla en la interacción conversacional. Desde 1975 el Análisis de la Conversación se ha extendido sobre un ancho campo, incluyendo temas nuevos importantes y profundizando en los ya tratados. Entre esos temas nuevos están las verbalizaciones y las actividades no verbales, es decir, elementos paralingüísticos y quinésicos relacionados con los mecanismos conversacionales o la estructuración interna de la interacción comunicativa (Cestero, 2000a).

Por otro lado, una parte de lo que hemos definido como comunicación no verbal, la correspondiente a determinados hábitos y costumbres culturales de una comunidad, es estudiada por historiadores o especialistas en folclore que nada o poco tienen que ver con la comunicación y su estudio.

Por último, existe algún especialista que en sus trabajos une los objetos de estudio particulares, la teoría y la metodología de distintas disciplinas mencionadas, como es el caso de F. Poyatos, cuya obra es una de las más influyentes en la actualidad en lo que respecta al estudio de la comunicación no verbal, en general, y a la aplicación de los conocimientos que sobre ella tenemos en la enseñanza de lenguas extranjeras, en particular.

Los estudios realizados hasta el momento no nos permiten conocer con todo detalle y en profundidad qué es la comunicación no verbal, cuáles son los signos y sistemas que la integran y cómo funcionan, pues la investigación sobre comunicación no verbal se encuentra aún en la fase de identificación, descripción y clasificación de signos y sistemas. Sin embargo, los conocimientos que sobre ella tenemos en la actualidad nos permiten estar seguros de que los signos no verbales constituyen una parte sustancial de la comunicación y de los medios de comunicación humanos. Tanto es así, que está bien establecido que para comunicar y comunicarnos utilizamos simultánea o alternativamente elementos verbales y no verbales y 
que, además, los signos verbales están en relación de dependencia con respecto a los signos no verbales (Poyatos, 1994a), por ello, para investigar la comunicación humana y enseñar a comunicar o comunicarse, se ha de prestar atención, conjuntamente, a los signos y sistemas verbales y a los signos y sistemas no verbales.

Tal y como la he definido al comienzo, la Comunicación no Verbal engloba todos los signos y sistemas de signos no lingüísticos que comunican o se utilizan para comunicar. Se incluyen, por tanto, dentro de ella los hábitos y las costumbres culturales en sentido amplio y los denominados sistemas de comunicación no verbal. Definida así, podemos establecer, por razones fundamentalmente prácticas, ya que no debemos olvidar que se trata siempre de elementos y aspectos interrelacionados, dos tipos diferentes de elementos constitutivos de lo que comúnmente se denomina comunicación no verbal:

A. Los signos y sistemas de signos culturales, es decir, el conjunto de hábitos de comportamiento y ambientales y las creencias de una comunidad que comunican, en sentido amplio y en sentido estricto ${ }^{2}$.

B. Los sistemas de comunicación no verbal, esto es, el conjunto de signos que constituyen los distintos sistemas de comunicación no verbal, a saber, el sistema paralingüístico, el quinésico, el proxémico y el cronémico.

Ambos contienen elementos universales y elementos peculiares de cada lengua y cultura y, por lo tanto, requieren estudio y enseñanza específicos. Pero, en esta ocasión, me voy a centrar en los sistemas de comunicación no verbal y, sobre todo, en los sistemas paralingüístico y quinésico, por ser los que están más claramente implicados en cualquier acto de comunicación oral humana, $y$, de forma bastante determinante, en algunos fenómenos característicamente discursivos como la ironía, a la que dedicaré una atención especial en las páginas que siguen.

\section{Los sistemas de comunicación no verbal}

El paralenguaje, la quinésica, la proxémica y la cronémica son los cuatro sistemas de comunicación no verbal reconocidos hasta el momento. De ellos, los dos primeros, uno fónico y otro corporal, son considerados sistemas básicos o primarios por su implicación directa en cualquier acto de comunicación humana, ya que se ponen en funcionamiento a la vez que el sistema verbal para producir cualquier enunciado ${ }^{3}$; los otros dos, el proxémico y el cronémico, son concebidos como sistemas secundarios o culturales, dado que actúan, generalmente, modificando o reforzando el significado de los elementos de los sistemas básicos ${ }^{4} \mathrm{o}$ independientemente, ofreciendo información social o cultural.

2 Se incluyen dentro de los sistemas de signos culturales tanto los grandes temas "tópicos", tales como sociedad, folclore, religión, política, historia, geografia, arte, etc., como los que podemos llamar "hábitos culturales menores". Estos últimos constituyen la verdadera esencia cotidiana; ejemplos de ellos son las creencias sobre el aspecto físico de las personas y todo lo que ello implica; las costumbres para decorar y habilitar lugares; el uso de utensilios y objetos; el comportamiento, el ambiente y las creencias en los establecimientos de distinto tipo públicos y privados o en los servicios públicos y privados. Véase a este respecto Cestero (1999a) y Poyatos (1994a).

3 De hecho, es imposible realizar un acto de comunicación únicamente con signos verbales, pues al emitir cualquier enunciado producimos, a la vez, signos no verbales paralingüísticos y quinésicos. Véase a este respecto la concepción y explicación de "la estructura triple básica de la comunicación humana" de Poyatos (1994a: capítulo 4).

4 Lenguaje, paralenguaje y quinésica. 
2.1. El sistema paralingüístico está formado por las cualidades y los modificadores fónicos, los indicadores sonoros de reacciones fisiológicas y emocionales, los elementos cuasi-léxicos y las pausas y silencios que a partir de su significado o de alguno de sus componentes inferenciales comunican o matizan el sentido de los enunciados verbales.

a) Las cualidades fisicas del sonido, tales como el tono, el timbre, la cantidad y la intensidad, y los modificadores fónicos o tipos de voz ${ }^{5}$ (dependientes del control que ejercemos sobre los órganos implicados en la articulación y la fonación) pueden aportar a cualquier expresión oral componentes inferenciales que, convencionalmente, pueden determinar la información que se desea dar o matizar el contenido o sentido de un enunciado o acto de comunicación. Así, por ejemplo, una expresión como "sé lo que quieres decir" puede comunicar entendimiento, desilusión, alegría o desprecio, dependiendo del tono con el que se emita o de la duración de algunos de sus sonidos, o puede tener distinto sentido si se dice con voz susurrante, con voz gritona o con voz temblorosa. Y un enunciado como "iqué bonito jersey lleva" puede resultar totalmente irónico si lo producimos con control de labios redondeados (tipo de voz) o si elevamos el tono y alargamos ciertos sonidos de los términos "bonito" y "jersey".

b) Algunas reacciones fisiológicas o emocionales, como la risa, el suspiro, el grito, la tos, el carraspeo, el bostezo, el llanto, el sollozo, el jadeo, el escupir, el eructo, el hipo, el estornudo, el castañeteo de dientes, etc. producen sonidos que contienen ciertos componentes inferenciales comunicativos que pueden variar de cultura a cultura ${ }^{6}$. Se trata de signos sonoros, emitidos consciente o inconscientemente, que tienen un gran rendimiento funcional. Sirva de ejemplo la risa, reacción emocional que manifiesta fundamentalmente alegría, pero que también puede expresar tristeza y miedo; además, la utilizamos como calificadora de enunciados, bien ajenos (indicando con ella acuerdo, desacuerdo, entendimiento, reconocimiento o seguimiento) o bien propios (marcando anécdotas y sucesos graciosos y suavizando errores, impropiedades conversacionales o enunciados comprometidos, como pueden ser los irónicos del tipo de "iqué rico estaba todo!" emitido con risa previa, riéndose o seguido de risa), y, por último, puede funcionar como señal de acción conversacional (indicando el comienzo y el final de los turnos o el seguimiento y la participación activa en una interacción $)^{7}$. También otros signos sonoros pueden conferir carácter irónico a un enunciado, ejemplo de ello son los siguientes: ((carraspeo)) o ((tos)) + "iqué bien habla!", ((bostezo $))+$ "iqué entretenido!".

c) Los elementos cuasi-léxicos son vocalizaciones y consonantizaciones convencionales de escaso contenido léxico, pero con gran valor funcional. Se consideran signos de este tipo gran parte de las interjecciones ( $j A h !, U y$ ! $; A j a !)$, las onomatopeyas (Mua-Mua, Pii-pii, Pon-pon, Miau, Croac), emisiones sonoras que cuentan con nombre propio (chistar, sisear, roncar, resoplar...) y otros muchos sonidos (Uff, Psi-psa, Hm, Iaj, Tl, Ajjj, Ouu...) que, sin tener un nombre o una grafia establecidos, se utilizan convencionalmente con un valor comunicativo similar al de determinados signos lingüísticos o quinésicos, de ahí que se conozcan como alternantes paralingüísticos ${ }^{8}$. A pesar de tratarse de elementos muy productivos, 
debido a la dificultad que entraña su identificación y su transcripción, no contamos aún con inventarios completos y detallados'; dichos inventarios habrán de hacerse atendiendo, por un lado, a la forma de los signos y, por otro, a su significado ${ }^{10}$.

La forma de los alternantes depende de su modo de producción, distinguiéndose entre signos vocálicos o vocalizaciones (AAaua para expresar cansancio o aburrimiento y Aja para indicar entendimiento), signos consonánticos o consonantizaciones (;MMh! para mostrar gran tamaño o $\mathrm{Hm}$ para indicar acuerdo o seguimiento) y signos combinados - vocalizaciones con consonantizaciones o viceversa- (Iiaj o Uuaj para comunicar que algo o alguien es feo o huele mal o $T s$ para iniciar discurso).

El significado de los alternantes depende de su aporte comunicativo. En este sentido cabe clasificarlos como:

a) Reguladores interactivos: $H m$ o $A$ ha de apoyo, eee de mantenimiento de turno, $T c$ de toma de palabra, Chss de llamada de atención, etc.

b) Referenciales, bien descriptivos, como $U u U u$ o UfF para indicar lejanía o Uuaj para aludir a una persona fea, o bien imitativos, como Mua-Mua para besar o Guau-guau para el ladrido del perro.

c) Expresivos: Aahhh o HJmm para mostrar bienestar físico o anímico, Brr para expresar impaciencia o nerviosismo...

d) Por último, hemos de recordar que la ausencia de sonido también comunica".

Las pausas ${ }^{12}$ tienen como función primordial regular el cambio de turno, indicando el final de uno y el posible comienzo de otro, en muchas lenguas y culturas, aunque la española no es una de las más típicas al respecto, debido al manejo tan particular que hacemos del mecanismo de alternancia de turnos en interacción (Cestero, 2000a). Pueden funcionar también como presentadoras de distintas clases de actos comunicativos verbales, tales como preguntas, narraciones o peticiones de apoyos, y, además, pueden ser reflexivas o fisiológicas, con las connotaciones que ello conlleva.

Los silencios ${ }^{13}$, por su parte, poco frecuentes en español, pueden ser confirmadores de enunciados previos o venir motivados por un fallo en los mecanismos interactivos tales como el de cambio de hablante, el de corrección y el de respuesta a pregunta o por un fallo comunicativo como en los casos de titubeos, dudas, reflexiones, etc.; además, pueden ser utilizados como presentadores de actos comunicativos (preguntas o narraciones), como enfatizadores del contenido de los enunciados emitidos o que se van a emitir, y, posiblemente, aunque no tenemos constancia de ello, como marcadores de fenómenos pragmático-discursivos como la ironía, el sarcasmo, el humor verbal o la metáfora.

2.2. El sistema quinésico está formado por los movimientos y las posturas corporales que comunican o matizan el significado de los enunciados verbales ${ }^{14}$, incluyéndose, además, dentro de él, aspectos tan relevantes como la mirada o el contacto corporal.

9 Uno de los estudios más completos sobre el tema es el de Poyatos (1993).

10 Véase Cestero (1999a) y Poyatos (1994b).

11 Para una mayor profundización sobre el tema véase Poyatos (1994b: 165-169).

12 Ausencia de habla durante un periodo de tiempo comprendido entre 0 y 1 segundo aproximadamente.

13 Ausencia de habla durante más de un segundo.

14 Para una mayor profundización en el tema véase Poyatos (1994b: capitulos 5-6). 
De forma general, pueden distinguirse tres categorías básicas de signos quinésicos: los gestos o movimientos faciales y corporales, las maneras o formas convencionales de realizar las acciones o los movimientos y las posturas o posiciones estáticas comunicativas, resultantes o no de la realización de ciertos movimientos. A pesar de que existe una relación de interdependencia entre las tres categorías, pues para describir un gesto es necesario mencionar la manera de realizarlo y la posición que adoptan los órganos ${ }^{15}$, sólo la primera ha sido tratada con cierta profundidad.

a) Los gestos son movimientos psicomusculares con valor comunicativo.

Las posibilidades de movimiento que ofrece el cuerpo humano son muchísimas, no obstante, podemos simplificarlas y distinguir dos tipos de gestos básicos que, generalmente, están interrelacionados, aunque pueden especializarse funcionalmente:

a. Gestos faciales, realizados, fundamentalmente, con los ojos, las cejas, el entrecejo y el ceño, la frente, los pómulos, la nariz, los labios, la boca y la barbilla, y

b. Gestos corporales, realizados, fundamentalmente, con la cabeza, los hombros, los brazos, las manos, los dedos, las caderas, las piernas y los pies.

Es difícil encontrar gestos simples producidos con un solo órgano, lo usual es poner en funcionamiento, en combinación y coestructuración, varios órganos a la vez; así, por ejemplo, cuando hacemos un saludo manual, a la vez que levantamos el brazo y agitamos la mano, elevamos, aunque sea ligeramente, las cejas, arrugamos la frente y esbozamos una sonrisa.

Cualquier acto discursivo oral conlleva, lógica y obviamente, gesticulación facial. En muchos casos, los gestos realizados son determinantes en la producción e interpretación del sentido, pero, en otras, no, su valor o función es secundario y la marcación fundamental proviene de signos gestuales corporales, paralingüísticos o lingüísticos. Al producir un acto comunicativo como /curvatura de boca hacia abajo//abertura considerable de ojos/ + " $j e s$ muy lista su niña!", los signos gestuales faciales pueden ser fundamentales para conferir carácter irónico al enunciado. Sin embargo, el mismo enunciado verbal puede emitirse elevando el tono, aumentando la intensidad o alargando algunos sonidos en el término "lista", o realizando, a la vez, un gesto de negación con la cabeza; en estos casos, serían signos paralingüísticos o gestuales corporales los marcadores fundamentales en la producción e interpretación del enunciado irónico, quedando como secundarios los signos gestuales faciales.

Huelga decir que la mirada es uno de los signos gestuales faciales más plurifuncionales que tenemos. Obsérvese cómo es suficiente con /bajar la mirada -dirigirla hacia el suelo, en transversal - /, para que el enunciado anterior (“¡es muy lista su niña!") tenga carácter irónico.

b) Las maneras son, como su propio nombre indica, las formas de hacer movimientos, tomar posturas $y$, en general, realizar actos no verbales comunicativos. Se refieren, por tanto, a las formas en que, convencionalmente, producimos gestos y posturas, por un lado, y a determinados hábitos de comportamiento culturales, por otro. La definición misma nos permite distinguir dos tipos de maneras básicas: en primer lugar, las maneras gestuales y posturales, que deben identificarse y describirse junto con el gesto o la postura mismos, ya que forman parte de su producción, $y$, en segundo lugar, las maneras de realizar hábitos de comportamiento culturales. 
c) Las posturas son las posiciones estáticas que adopta o puede adoptar el cuerpo humano y que comunican, activa o pasivamente. Como en el caso de las maneras, son signos no verbales que, por un lado, forman parte de los gestos mismos, pues su significado puede variar dependiendo de la postura final que adoptan los órganos implicados, $y$, por otro, funcionan como signos comunicativos independientes, como es el caso de estar, por ejemplo, de pie, con los brazos arqueados y las manos apoyadas en la cintura (/en jarras/) o sentados, con las piernas ligeramente dobladas y las manos unidas en el regazo.

Este tipo de signos también puede ser utilizado para conferir carácter irónico a un enunciado. No hay más que imaginar la producción del siguiente ejemplo: "Es como yo, muy fina..." /sentada, con las piernas considerablemente abiertas/. En estos casos, como en el ejemplo anterior de /negación con la cabeza/, lo que hacemos es utilizar el valor comunicativo de un signo no verbal (una postura, un gesto...) que contradice el significado del enunciado verbal para conseguir un efecto determinado: atenuación de crítica o descortesía mediante ironía.

2.3. El sistema proxémico está conformado por los hábitos relativos al comportamiento, al ambiente y a las creencias de una comunidad que tienen que ver con la concepción, el uso y la distribución del espacio y con las distancias culturales que mantienen las personas en interacción ${ }^{16}$. Se consideran signos proxémicos culturales, por ejemplo, la distribución que del espacio natural hace el ser humano (configuración de ciudades y pueblos, de calles, de bosques, de parques, de casas, de mobiliario...), el comportamiento de las personas con respecto al uso del espacio (el orden y desorden en la colocación de objetos, el mantenimiento de filas, el respeto de espacios prohibidos o privados...) y, en relación con ellos, se estudia dentro de la proxémica el sentido cultural de intrusión o violación de la territorialidad y la privacidad.

Es de especial importancia la llamada proxémica interaccional, a través de la cual se establecen las distancias a las que las personas de una comunidad realizan distintas actividades interactivas o comunicativas, tales como consolar, conversar, mantener entrevistas laborales, impartir clases o dar conferencias; estas distancias varían transculturalmente, de la misma manera que la importancia que se le confiere al hecho de guardarla o no ${ }^{17}$. Y dentro de la proxémica interaccional está comprendida una serie de signos no verbales que, convencionalmente, pueden modificar o reforzar el significado de otros signos comunicativos verbales o no verbales o sustituirlos con completo valor significativo. Por ejemplo, si \{nos aproximamos a nuestro interlocutor mientras le decimos "quiero ir contigo", estamos confirmando o reforzando el sentido del enunciado verbal emitido y podemos utilizar una \{separación brusca $\}$ de una persona para indicar que la rechazamos o que no aprobamos lo que dice, o nos podemos \{acercar\} a nuestro interlocutor para cederle la palabra y pedirle que hable.

16 Para una mayor profundización en el tema véase Hall (1963 y 1966), Poyatos (1975 y 1976) y Watson (1970).

17 Así, en las llamadas culturas de contacto, de las que forma parte la española, las distancias que se mantienen entre las personas que interactúan suelen ser menores que las que se guardan en las culturas que no son de contacto, por ejemplo, la distancia que mantienen los españoles mientras conversan puede ser de unos 50 ó 75 centímetros, lo que para un alemán o un noruego supondría una invasión del espacio propio, por tratarse de una distancia demasiado reducida para el tipo de actividad que se realiza. 
2.4. El tiempo también comunica, bien pasivamente, ofreciendo información cultural, bien activamente, modificando o reforzando el significado de los elementos del resto de sistemas de comunicación humana. Su estudio se ha denominado cronémica, que se define como la concepción, la estructuración y el uso que hace del tiempo el ser humano ${ }^{18}$.

En general puede hablarse de tres tipos de categorías dentro del estudio del tiempo: el tiempo conceptual, el tiempo social y el tiempo interactivo ${ }^{10}$.

Forman parte del llamado tiempo conceptual los hábitos de comportamiento y las creencias relacionados con el concepto que tienen del tiempo las distintas culturas, por ejemplo, si lo valoran o no o si lo consideran como algo concreto o abstracto, material y tangible o intangible, y por qué; también los relacionados con la distribución del tiempo que hacen las distintas comunidades y con la incidencia del tiempo en la acción humana, tales como la planificación del tiempo o la realización usual de una o varias actividades a la vez; asimismo, se atiende en esta categoría al valor cultural que tienen distintos conceptos como puntualidad e impuntualidad, prontitud y tardanza, un momento, un rato, mucho tiempo y una eternidad.

El tiempo social, que depende de forma directa del tiempo conceptual, está constituido por los signos culturales que muestran el manejo del tiempo en las relaciones sociales; se trabaja dentro de él, por ejemplo, la duración de determinados encuentros sociales como reuniones, entrevistas de trabajo o visitas, la estructuración de las actividades diarias tales como desayunar, comer, merendar y cenar o los momentos del día apropiados para determinadas actividades sociales.

Por último, se incluye dentro del tiempo interactivo la mayor o menor duración de signos de otros sistemas de comunicación que tiene valor informativo, bien porque refuerza el significado de sus elementos o bien porque especifica o cambia su sentido. Así, son signos cronémicos, por ejemplo, la mayor o menor duración de los sonidos de algunas palabras, de algún gesto o de las pausas, con las connotaciones que conllevan, o el aumento de velocidad en la emisión de un enunciado como "no puedes hacerlo así, tienes que hacerlo...", que disminuye su efecto crítico o corrector.

\section{Los signos no verbales en el proceso de comunicación humana}

Los elementos que conforman los sistemas de comunicación no verbal, especialmente el paralingüístico y el quinésico, se utilizan de forma simultánea con los elementos del sistema verbal, o alternando con ellos, en cualquier acto de comunicación humana. Es únicamente en la combinación de los significados o valores de todos los signos (verbales y no verbales) emitidos donde se encuentra el contenido o sentido de cada enunciado, por lo que los elementos que integran los sistemas de comunicación no verbal, de la misma manera que los que componen el sistema verbal, precisan una investigación específica y completa.

Los signos que ahora nos ocupan serán más o menos relevantes en el proceso de comunicación dependiendo del uso que de ellos se haga. A este respecto cabe decir que la gran mayoría de los elementos paralingüísticos y quinésicos (también los proxémicos y cronémi- 
$\cos$, pero en menor medida) son plurifuncionales y pueden cumplir, en cualquier momento de la interacción, una o más de las siguientes funciones fundamentales:

1) Añadir información al contenido o sentido de un enunciado verbal o matizarlo.

Es de todos bien conocida la relación de dependencia que existe entre el sistema verbal y los sistemas no verbales, tanto es así que es imposible comunicar verbalmente sin emitir, a la vez, signos no verbales paralingüísticos y quinésicos que añaden información al contenido o sentido de un enunciado verbal o lo matizan. Ya se ha mencionado con anterioridad que la comunicación humana se realiza poniendo en funcionamiento, al menos, signos de tres sistemas distintos; es lo que Poyatos (1994a: capítulo 4) denomina la "estructura triple básica", de la que forman parte, de manera obligatoria, el lenguaje, el paralenguaje y la quinésica; dicha estructura triple básica puede ser modificada o matizada, a su vez, por signos proxémicos y cronémicos, $\mathrm{y}$, por supuesto, todo el proceso está inserto en una cultura que lo determina.

Cuando se utilizan signos paralingüísticos, quinésicos, proxémicos o cronémicos para añadir información al contenido o sentido de un enunciado verbal o matizarlo, pueden cumplir distintas subfunciones, a saber:

1.- Especificar el contenido o sentido de un enunciado verbal. El tono, la intensidad o la mayor duración de algunos sonidos al emitir un "claro" o un "seguro" especificarán si se trata de un enunciado de acuerdo, de asentimiento o, incluso, de desconformidad. El tipo de voz o los gestos faciales con los que produzcamos un enunciado como "por fin estás aquí" comunicarán si nos sentimos contentos, desilusionados, temerosos o enfadados por el hecho de que el interlocutor haya venido.

2.- Confirmar el contenido o sentido de un enunciado verbal. Por ejemplo, un gesto de negación o refutación que acompaña a un "no me gusta" verbal, una elevación tonal y un /fruncimiento de entrecejo/ al emitir "es muy feo" o una sonrisa amplia que confirma el sentido de una expresión como "me encanta" referida a un regalo. 3.- Reforzar el contenido o sentido de un enunciado verbal. Así, un tono elevado o una voz gritona que acompaña a un "eso no se hace" refuerza el sentido del enunciado verbal, de la misma manera que el aumento de velocidad de emisión al decir "no lo entiendo" o un fuerte abrazo a la vez que se dice "me alegro de verte".

4.- Debilitar el contenido o sentido de un enunciado verbal. Por ejemplo, al expresar sonriente un enunciado del tipo "esto no es así, es..." o al bajar el tono al decir "podría hacerse de otra manera" cuando se muestra desconformidad con el interlocutor.

5.- Contradecir el contenido o sentido de un enunciado verbal. Así, por ejemplo, al decir en tono alto, casi gritando, y con el ceño fruncido "me parece una idea fantástica", lo que comunicamos, en realidad, es que no nos gusta la idea propuesta por nuestro interlocutor, o cuando producimos un "sí" con determinados gestos faciales, lo que comunicamos es "no".

6.- Camuflar el verdadero sentido de un enunciado verbal. Por ejemplo, al decir en tono bajo y en velocidad lenta de emisión, o con determinados gestos faciales, un enunciado como "no me importa que no mencionara nada de la fiesta", podemos estar intentando camuflar nuestros verdaderos sentimientos.

Teniendo en cuenta el alcance de estos usos de los signos no verbales, es fácil aceptar, sin reparos, la existencia de la triple estructura básica a la que antes hacía referencia y, 
concebido así el proceso de comunicación humana, cabe preguntarse si puede hablarse con propiedad de comunicación lingüística o si, por el contrario, lo que ha de investigarse y describirse es la expresión lingüística, paralingüística y quinésica conjuntamente, ya que, irremediablemente, en cualquier acto comunicativo se ponen en funcionamiento signos del sistema verbal y de los sistemas no verbales a la vez.

2) Comunicar, sustituyendo al lenguaje verbal.

Como se ha argumentado previamente, los elementos del sistema verbal, obligatoriamente, deben ir acompañados de elementos de los sistemas paralingüístico y quinésico para que se produzca comunicación, sin embargo, no ocurre lo mismo con los signos no verbales, pues algunos de ellos pueden alternar con signos verbales en un mismo acto comunicativo o utilizarse en lugar de ellos, siendo en muchas ocasiones más significativos. Así, se puede comunicar el deseo de que alguien se vaya de distintas maneras: bien con un enunciado coestructurado verbal-no verbal ("vete"/vete/), bien con un enunciado en el que alternen signos verbales y no verbales ("Oye, Pedro" + gesto para /vete/) o bien con la producción de un signo no verbal paralingüístico ((HeeE) - (FusFus)), un signo no verbal quinésico (gesto para /vete/) o una coestructuración formada por un signo paralingüístico y quinésico a la vez ((FusFus) /vete/). De la misma manera, se puede comunicar que se tiene sueño o frío, que se está hambriento o sediento y que estamos felices, temerosos, aburridos o nerviosos mediante la utilización simultánea o alternante de signos verbales, paralingüísticos y quinésicos o usando sólo signos de los sistemas de comunicación no verbal.

3) Regular la interacción.

Son muchos los elementos de los sistemas no verbales que sirven para regular, organizar o estructurar la interacción, es más, habitualmente cualquier actividad interactiva se regula y estructura a través de ellos. Se trata de una función de gran relevancia, pues los fallos en la regulación suelen provocar la interrupción de la comunicación. Son ejemplos de elementos paralingǘsticos o quinésicos utilizados frecuentemente con esta función: el descenso tonal, la pausa, la fijación de mirada en el interlocutor o el alargamiento de sonidos finales para distribuir el turno de palabra; la sonrisa, el asentimiento con la cabeza, los elementos cuasiléxicos del tipo de $(h \mathrm{~h}),(a h a),(a h) \ldots$ para apoyar; los titubeos, clics y aspiraciones para tomar la palabra, y la reorganización postural o la dirección de la mirada para mantener o ceder la palabra.

4) Subsanar deficiencias verbales.

Solemos utilizar determinados signos de los sistemas de comunicación no verbal, además, para evitar los vacíos conversacionales o discursivos producidos por deficiencias verbales momentáneas o por desconocimiento de los elementos correspondientes del sistema lingüístico. Signos paralingüísticos como $(E e),(M m)$ o $(H h)$ llenan los vacíos provocados por titubeo o duda y un gesto manual ilustrativo puede sustituir al elemento léxico que no conocemos o no recordamos en un momento dado de la comunicación.

5) Intervenir en conversaciones simultáneas.

Por último, gracias a los sistemas de comunicación no verbal podemos mantener más de una conversación a la vez y expresar más de un enunciado de forma simultánea, sirvan de ejemplo esas conversaciones que mantenemos con los que nos rodean cuando hablamos por teléfono o ese estar en dos conversaciones a la vez, escuchando al que habla y comentando lo que dice con el resto de interlocutores mediante señales hechas con los pies, las manos y la mirada. 
No puedo terminar este breve repaso de la importancia de los signos no verbales en el proceso de comunicación humana, sin aludir a algunas de sus características más importantes: los elementos que nos ocupan pueden comunicar activa o pasivamente, es decir, pueden ser utilizados para comunicar, pero también pueden comunicar sin que lo provoquemos o deseemos. En estrecha relación con esta característica está la utilización consciente e inconsciente de los signos no verbales: es posible y frecuente que utilicemos de forma inconsciente signos no verbales que realicen actos de comunicación imperceptibles para el emisor, pero no para el receptor, que les dará más crédito incluso que a los signos verbales por tratarse, precisamente, de actos involuntarios ${ }^{20}$.

Las características y funciones de los signos no verbales que acabo de mencionar permiten afirmar que, si nuestro deseo es conocer el proceso de comunicación humana y su producto, lo más apropiado es abordar su estudio teniendo en cuenta todos los signos y factores que inciden en él. En este sentido, es preciso atender no sólo al sistema verbal, sino también, y conjuntamente, a los sistemas no verbales, pues, sólo así podremos analizar y explicar con propiedad los actos de comunicación en todas sus dimensiones.

\section{El estudio de los signos no verbales}

Como he apuntado al comienzo de estas páginas, los estudios sobre comunicación no verbal se encuentran aún en la fase de identificación, descripción y clasificación de signos y sistemas, pero los conocimientos que sobre ella tenemos en la actualidad revelan la necesidad de empezar a incluirla en la investigación sobre comunicación humana, especialmente en aquella que se centre en el discurso o la interacción oral. Ahora bien, la forma de abordar el estudio de los signos y sistemas de comunicación no verbal dependerá sustancialmente del objetivo último de nuestra investigación, esto es, de nuestro objeto de estudio y del fin que nos ha llevado a analizarlo. Requerirán un tratamiento diferente los signos que se usan para realizar un acto completo de comunicación o para regular la interacción y los signos que matizan o especifican el contenido o sentido de un enunciado. Los primeros se pueden tratar de forma aislada, describiendo e inventariando los elementos y explicando su función, pero los segundos exigen un estudio detallado de la implicación de los signos de los distintos sistemas en la consecución del sentido del enunciado. Y, metodológicamente, serán diferentes, también, los proyectos de investigación que tengan un carácter aplicado y los que presenten un carácter teórico o descriptivo.

\subsection{Elaboración de inventarios de signos no verbales}

En varias ocasiones he tratado el estudio de los signos no verbales para su aplicación a la enseñanza y el aprendizaje de lenguas extranjeras ${ }^{21}$. En tal contexto, en el que se trabaja,

\footnotetext{
20 Sirvan de ejemplo los clics, las aspiraciones o los cambios posturales que realizamos cuando deseamos hablar y nuestro interlocutor no nos cede la palabra y que suelen ser involuntarios o tan espontáneos que el emisor no se da cuenta de su producción, pero para el interlocutor constituyen una señal inequívoca de petición de palabra; lo mismo ocurre con los gestos manuales que hacemos cuando estamos nerviosos, con la desviación de la mirada o con la no emisión de apoyos conversacionales cuando no estamos interesados en un tema, con la aproximación corporal a la persona que nos agrada...
}

21 Cestero (1999a, 1999b, 2000b y 2004). 
fundamentalmente, con signos que comunican sustituyendo al lenguaje verbal, regulan la interacción o subsanan deficiencias verbales ${ }^{22}$, es necesario elaborar inventarios de signos no verbales y realizar estudios comparativos interculturales o inter-comunitarios que permitan seleccionar los elementos peculiares de cada cultura (Cestero, 1999a y 1999b, y Poyatos, 1994b: 227-233). Un método de trabajo para la realización de inventarios de signos no verbales homogéneos, completos y detallados, siguiendo las cuatro fases propias de la investigación empírica, puede ser el siguiente:

1) Selección y constatación del objeto de estudio

Lo primero que debemos establecer, y muy claramente, es nuestro objeto de estudio, que, en el caso de la comunicación no verbal, tendrá que ver con el sistema sobre el que pretendemos trabajar y con las categorías que vamos a investigar. Una vez seleccionado el objeto de estudio, se ha de realizar una clasificación provisional de los signos pertenecientes a la categoría y al sistema elegidos, recurriendo a la bibliografía existente y a nuestros propios conocimientos sobre el tema.

Cuando los inventarios son específicos para la enseñanza de lenguas extranjeras, lo más conveniente es trabajar con los distintos sistemas no verbales, de forma que la inclusión de los signos no verbales en los programas de enseñanza pueda ser integrada. La clasificación más apropiada, atendiendo a los diseños curriculares más actuales, es la nocio-funcional y se realizará a partir de las funciones básicas de los signos, que aparecerán, así, distribuidos, por un lado, según sus usos principales ${ }^{23} \mathrm{y}$, por otro, según su valor funcional o el significado de su alternante o correspondiente lingüístico ${ }^{24}$.

2) Recogida del material

La clasificación provisional de los elementos del sistema de comunicación no verbal con el que deseamos trabajar o de los usos funcionales básicos que deseamos inventariar, nos sirve de guía para realizar la recogida de materiales con el fin de obtener un corpus amplio para analizar. Dado que la percepción y recolección de los signos no verbales entraña gran

22 Esto no quiere decir que no haya que tratar los signos que matizan o especifican el contenido de los enunciados verbales o no verbales cuando se enseñan lenguas extranjeras, pero se trabajan en niveles superiores, por su complejidad, y no se incluyen en los inventarios convencionales.

23 Signos no verbales con usos sociales, signos no verbales con usos estructuradores del discurso y signos no verbales con usos comunicativos.

24 Reelaborando los contenidos funcionales recogidos por el Instituto Cervantes (1994), puede servir de ejemplo la siguiente clasificación básica (Cestero, 1999b):

Signos no verbales con usos sociales: saludar; despedirse; presentar y reaccionar al ser presentado; pedir perdón; dar las gracias; felicitar; pedir y conceder permiso, y ofrecer o invitar y reaccionar a ello.

- Signos no verbales con usos estructuradores del discurso: dirigirse a alguien; iniciar o finalizar el discurso; indicar que se toma, se mantiene o se cede el turno de palabra, o instar al interlocutor a que lo haga; conectar elementos o partes del discurso; señalar que no se ha entendido algo de lo emitido y pedir su repetición; pedir que se hable más alto, más bajo o más despacio; comprobar e indicar que se está siguiendo la comunicación; señalar correcciones, clarificaciones o reformulaciones del discurso propio o del ajeno; marcar la caracterización de una interrupción, y señalar la producción, la necesidad o el deseo de cambio temático.

- Signos no verbales con usos comunicativos: identificar objetos y personas; ubicar objetos, personas, sucesos y lugares; describir estados, personas, animales, lugares, objetos y cosas; referirse a acciones presentes o habituales, pasadas y futuras; opinar; expresar conocimiento, grado de certeza, sentimientos, sensaciones y deseos; sugerir, recomendar o aconsejar y reaccionar a ello; mandar o pedir a otros que hagan algo; dar instrucciones, y prevenir, advertir o animar. 
dificultad, recomiendo cuatro procedimientos diferentes de recogida que deben realizarse de forma sucesiva:

2.1. Introspección. El investigador, a través de su propia introspección, realizará el primer inventario de signos no verbales y la descripción detallada de cada uno de ellos.

2.2. Observación. Posteriormente, el investigador observará, participativamente, la forma en que otros miembros de la comunidad utilizan los signos recogidos previamente en el inventario. Esta labor servirá para constatar que los elementos inventariados son generales y que su descripción es la correcta.

2.3. Realización de encuestas. Los elementos de los sistemas de comunicación no verbal, igual que ocurre con los del sistema verbal, varían dependiendo de la caracterización social de la persona que los utiliza, de su origen geográfico o de la situación en que son usados. Por ello, es recomendable la realización de encuestas sociolingüísticas o dialectales, en las que se controlen factores como el sexo, la edad, el nivel sociocultural, el origen de las personas o el contexto de uso, con objeto de constatar la variación de los signos no verbales. Dichas encuestas deben ser grabadas íntegramente, tanto si son orales como si están basadas en cuestionarios escritos, y, en este último caso, debe ser el propio investigador el que tome los datos, nunca el informante, pues la descripción de los signos no verbales es una tarea difícil, que debe hacerse de forma sistemática, y que resultaría tediosa, si no imposible, para cualquier persona que no conozca o tenga cierta técnica y experiencia.

2.4. Revisión de la comunicación no verbal en programas de televisión, en prensa, en obras literarias... Como última fase de recogida de material es conveniente la revisión de la utilización de los signos estudiados en programas de televisión de corte costumbrista, en las ilustraciones o entrevistas periodísticas, en cómics, en acotaciones o comentarios de autor en obras literarias ${ }^{25}$, etc., ya que se trata de diferentes medios de comunicación en los que podemos encontrar una gran cantidad de usos y personas diferentes que nos servirán para constatar, una vez más, los datos obtenidos previamente.

3) Análisis del material

En el tipo de investigación que aquí tratamos, el análisis cualitativo del material recogido se va realizando de forma progresiva a la vez que se efectúan las distintas fases de recolección de datos. Por ello, esta tercera fase tiene como objetivo establecer la clasificación definitiva de los elementos estudiados y su forma habitual de uso y producción, así como las variantes sociales, geográficas o situacionales que aparecen de forma sistemática, a partir de la realización de análisis cuantitativos.

4) Presentación de los resultados

Los resultados del estudio realizado deben presentarse en forma de inventarios en los que se recojan, clara y sistemáticamente, todos los elementos trabajados y se establezcan las relaciones pertinentes entre ellos. En los inventarios dirigidos de forma especial a la enseñanza de lenguas extranjeras, los signos no verbales paralingüísticos, quinésicos, proxémicos y cronémicos deben aparecer juntos, pues juntos han de enseñarse, clasificados, como antes 
hemos dicho, por funciones comunicativas básicas y, además, han de combinarse con los signos o el conjunto de signos verbales que se utilizan para realizar la misma función.

Una vez realizados los inventarios específicos, se puede comenzar, de forma progresiva, a integrar los elementos de los distintos repertorios en los diseños curriculares ${ }^{26}$.

Este método de investigación es válido, también, para llevar a cabo estudios teóricodescriptivos de distinto tipo (conducentes a repertorios, clasificaciones, etc.), pero no es adecuado cuando nuestro objeto de estudio no son los signos no verbales en sí mismos, sino la incidencia que tienen en el contenido o sentido de enunciados verbales-no verbales o en determinados actos de comunicación, aunque los resultados de investigaciones conducentes a la elaboración de inventarios o a la descripción de signos no verbales pueden servir de base para este otro tipo de trabajos.

\subsection{Estudio de la incidencia de los signos no verbales en la matización o especificación del sentido o contenido de enunciados o actos de comunicación}

He destacado con anterioridad la relación de dependencia que existe entre el sistema verbal y los sistemas no verbales, aludiendo, incluso, a la imposibilidad de comunicar verbalmente sin emitir, a la vez, signos no verbales que añaden información al contenido o sentido de un enunciado verbal o lo matizan, ya que la comunicación humana se realiza poniendo en funcionamiento, al menos, signos de tres sistemas distintos: lingüístico, paralingüístico y quinésico. Este hecho, fundamental en cualquier acto de comunicación humana, se comprueba fácilmente en determinados fenómenos pragmático-discursivos como la ironía, pues, sea cual sea su concepción ${ }^{27}$, en su producción, reconocimiento e interpretación están implicados signos no verbales, si bien en unas ocasiones su incidencia es más determinante que en otras ${ }^{28}$.

La ironía es un fenómeno pragmático-discursivo que se consigue mediante la utilización e interpretación de indicadores lingüísticos, de marcadores no verbales o de una combinación de elementos de los dos tipos. Tanto es así, que, en principio, cualquier enunciado puede ser irónico si el hablante lo pretende, y, para ello, sólo tiene que utilizar un indicador

26 Véanse Cestero (1999a y 2000), Hurley (1992), Melrose (1992) y Poyatos (1992, 1994b y 2006).

27 En la bibliografía de corte pragmático encontramos distintas concepciones, y consecuentemente diferentes tratamientos, de la ironía verbal. Destacan, al respecto, la postura de Grice (1975 y 1978) y sus seguidores, que consideran la ironia como violación convencional de la máxima de cualidad, la de Sperber y Wilson y los relevantistas (Sperber y Wilson, 1978 y 1981; Torres, 1999 y Reyes, 2002), que conciben los enunciados irónicos como "menciones ecoicas" de otros enunciados que los deforman o contradicen, o la asumida por el grupo GRIALE, que trata el sentido irónico como violación convencional del requisito de cualidad, entendido éste como principio cooperativo básico en cualquier acto comunicativo, lo que, a su vez, genera una inversión del resto de principios conversacionales (Ruiz, Marimón, Padilla y Timofeeva, 2004 y http//griale.ua.es).

28 Son varios ya los investigadores que han apuntado la utilización e importancia de los indicadores o marcadores -para ser más precisos utilizando terminología de lingüistica bien establecida- de ironización. Entre ellos está el grupo GRLALE de la Universidad de Alicante, que está llevando a cabo, en la actualidad, un proyecto de investigación cuyo objetivo es el análisis pragmático de la ironía verbal con vistas a su clasificación y aplicación a la enseñanza de español como lengua extranjera. GRIALE parte, en sus estudios, de que la caracterización de un enunciado como irónico depende de la utilización de indicadores lingüísticos, paralingüísticos y acústico-melódj$\cos$, e intenta identificarlos y analizar su funcionamiento. Se trata, sin duda, de un proyecto interesantísimo, que puede dar mucha información sobre el fenómeno conocido como ironía, en general, y sobre el funcionamiento y uso de los signos no verbales, en particular. 
o marcador de ironización. Piénsese, por ejemplo, en un enunciado tan simple como "me voy a comer" que, emitido con determinada gesticulación facial, con una sonrisa específica o con signos paralingüísticos caracteríscos - tono alto, intensidad fuerte o alargamiento de algunos sonidos- puede presentar sentido irónico.

Los indicadores a los que acabo de aludir suelen considerarse de tipo no verbal, aunque ello entraña un problema de fondo o perspectiva con respecto al componente entonativo (acústico-melódico), que tradicionalmente se ha tratado como parte del sistema verbal-lingǘstico ${ }^{29}$, pero, en los estudios de comunicación no verbal, se considera como un signo no verbal paralingüístico siempre que incida, de forma determinante (y parece hacerlo cuando varía de forma ostensible con respecto a los parámetros normales de uso - presentando, entonces, distintas funciones expresivas, actitudinales, situacionales...-), en el significado o sentido de las expresiones verbales ${ }^{30}$. A tenor de lo que acabo de mencionar, y en consonancia con la clasificación de los signos no verbales que he presentado antes, el estudio de la ironía debería partir de la existencia de dos tipos de indicadores o marcadores: lingüísticos y no verbales, subclasificables, a su vez, en distintas clases y categorías. Con respecto a los indicadores no verbales, que son los que aquí nos ocupan, podrían distinguirse:

1.- Indicadores o marcadores paralingüísticos.

A. Cualidades y modificadores fónicos:

* tono, timbre, duración e intensidad,

* esquemas entonativos y

* tipos de voz.

B. Sonidos característicos de reacciones fisiológicas o emocionales: risa, carraspeo, tos, suspiro, bostezo, etc. emitidos antes de discurso verbal, después de discurso verbal o durante discurso verbal.

C. Elementos cuasi-léxicos.

D. Pausas y silencios.

2.- Indicadores o marcadores quinésicos.

A. Gestos

a. gestos faciales, con especial atención a la sonrisa y a la mirada, y

b. gestos corporales

B. Maneras, si las hay.

C. Posturas

3.- Indicadores o marcadores proxémicos.

4.- Indicadores o marcadores cronémicos.

Por otro lado, de las subfunciones que pueden cumplir los signos no verbales cuando se utilizan para añadir información al contenido o sentido de un enunciado verbal o matizarlo, cuatro, al menos, parecen estar directamente relacionadas con la ironía: especificar el contenido o sentido de un enunciado verbal, confirmar el contenido o sentido de un enunciado verbal, reforzar el contenido o sentido de un enunciado verbal y contradecir el contenido o sentido de un enunciado verbal. En la primera y la última (especificar y contradecir el contenido o sentido de un enunciado), los signos no verbales juegan un papel determinante

29 No hay más que releer, para apreciarlo, a modo de ejemplo, los ya clásicos trabajos de Navarro Tomás (1985, $22^{a}$ ed.) o Quilis (1993).

30 Poyatos (1994b: cap. 1). 
en la producción e interpretación del enunciado o acto de comunicación irónico, mientras que, en la segunda y tercera (confirmar o reforzar), su incidencia es secundaria, pues pueden no resultar imprescindibles para la creación e interpretación del sentido irónico de un enunciado o acto de comunicación. Así, en el momento de analizar la ironía, debemos tener en cuenta, también, si los signos no verbales son indicadores o marcadores fundamentales o secundarios en la producción e interpretación del enunciado irónico, sin perder de vista que ello podría ser únicamente contextual, es decir, su incidencia fundamental o secundaria podría depender del contexto de uso, no del tipo de elemento utilizado. No obstante, no tendremos certeza de ello hasta que se aborde el estudio empírico de los indicadores no verbales y su funcionamiento en los enunciados o actos de comunicación irónicos.

Como puede apreciarse por lo expuesto hasta aquí, lo único que puedo ofrecer, a partir del estado actual de los estudios de comunicación no verbal, son algunos supuestos básicos que han de tenerse en cuenta al organizar el procedimiento de recogida, preparación y análisis de materiales para el estudio de la ironía. Serán los resultados de la investigación los que nos permitan conocer, en detalle y profundidad, cuáles son los tipos de signos - lingüísticos o no verbales- que intervienen en la producción e interpretación de los enunciados o actos de comunicación irónicos (es decir, cuáles son los indicadores lingüísticos y no verbales de la ironía) y cuál es la incidencia de los indicadores en la producción e interpretación del sentido irónico de un enunciado (esto es, si son fundamentales o secundarios), y serán los resultados los que nos permitan establecer tipologías y clasificaciones definitivas de indicadores de la ironía, teniendo en cuenta, para ello, frecuencias de uso.

El procedimiento metodológico que, bajo mi punto de vista, sería idóneo para trabajar este tema o fenómenos similares es el que tiene en cuenta las cuatro fases propias de investigación empírica que apunto a continuación:

1) Recogida de materiales

El estudio de fenómenos discursivos o pragmáticos como la ironia ha de hacerse sobre emisiones reales producidas de forma natural, lo que hace obligatoria una recogida especifica de datos.

Dar con la técnica de recogida de materiales apropiada para este tipo de investigación constituye un reto. Deberemos decidir, antes de comenzar, si trabajaremos con corpora de textos escritos u orales. Los textos escritos nos pueden ser útiles para analizar los indicadores lingüísticos, pero no los no verbales (a excepción de los textos literarios que presentan acotaciones del autor al respecto, aunque están basadas en la introspección y, por lo tanto, la fiabilidad puede no ser la deseada), por ello, el tipo de fenómeno que se pretende estudiar parece requerir, más bien, una investigación sobre corpora orales. A este respecto, y dado que en la caracterización de un enunciado como irónico pueden incidir marcadores verbales o no verbales, parece obvio que las grabaciones de materiales deben ser hechas en soporte audio-visual, pues aunque el indicador fundamental de un enunciado irónico pudiera ser lingüístico o paralingüístico (y, por lo tanto, audible), es conveniente el análisis completo del enunciado, ya que podríamos estar ante una combinación de signos lingüísticos-paralingüísticos-gestuales fundamental o ante la utilización de inđicadores lingüísticos o paralinguísticos fundamentales matizados por marcadores gestuales secundarios.

Por otro lado, tengo la impresión de que los enunciados irónicos no presentan una frecuencia de aparición lo suficientemente alta en conversación, interacción o discurso como para obtener, de un corpus razonable, en cuanto a tamaño, la cantidad y la variedad de casos 
necesarias para realizar un análisis amplio y detallado. Esto hace conveniente la utilización de alguna técnica suplementaria que nos permita recoger gran cantidad de material para contrastar y complementar los datos procedentes del análisis de grabaciones. Quizás se podría elaborar una especie de cuestionario, con enunciados contextualizados de distinto tipo, y aplicarlo, controlando variables sociales, en forma de encuesta. Lógicamente, la entrevista debería ser grabada, en vídeo, íntegramente.

2) Transcripción de los materiales.

En el caso de que trabajemos con datos orales, una vez recogido el material, debe procederse a su transliteración o transcripción, con objeto de preparar los datos adecuadamente para la codificación y el análisis. La transliteración de los signos lingüísticos se suele hacer en ortografía ordinaria, utilizando algunas convenciones (muy pocas para que no dificulten la lectura y comprensión) de uso bastante generalizado ${ }^{3 !}$.

La transcripción o representación de los signos no verbales es mucho más complicada, pues no existe un sistema de convenciones de uso común y para la mayoría de los signos no tenemos ni siquiera descripciones que nos faciliten la creación de convenciones adecuadas.

Fernando Poyatos (1993 y 1994b: 47-48) ha ofrecido una lista bastante extensa de símbolos para recoger signos paralingüísticos que podría ser útil en este tipo de estudios, siempre que se complete atendiendo a las necesidades que vayan surgiendo. Reproduzco, a continuación, los más aprovechables:

Marcadores paralingüísticos

A. Cualidades y modificadores fónicos:
* tono: $(\downarrow \downarrow)$ muy bajo;
$(\downarrow)$ bajo;
$(\uparrow)$ alto;
$(\uparrow \uparrow)$ muy alto
* timbre:
(| 2) muy bajo;
(| 1) bajo;
(1 |) alto;
(2 |) muy alto
* duración:

* intensidad:

(") hiperacortamiento; (") acortamiento;

(:) alargamiento simple; (:::) hiperalargamiento

(= $)$ muy baja/suave; $\quad(\mid++)$ muy alta/fuerte

(- $\mid$ ) baja/suave; $\quad(\mid+)$ alta/fuerte

* esquemas entonativos: pueden utilizarse los signos que recojo a continuación, acompañados de los fenómenos o aspectos entonativos específicos de cada enunciado o grupo melódico: $(\boldsymbol{\nearrow})$ entonación ascendente; $(\boldsymbol{\nabla})$ entonación descendente; $\rightarrow$ ) entonación suspendida

* tipos de voz: puede indicarse el tipo de voz entre doble paréntesis (( )), especificando su denominación ((voz chillona)) o sus órganos de control ((voz con labios redondeados)).

B. Sonidos característicos de reacciones fisiológicas o emocionales como risa, carraspeo, tos, suspiro, bostezo, etc., emitidos antes de discurso verbal, después de

31 Pueden servir de ejemplo las que habitualmente utiliza el grupo VA.LES.CO en sus publicaciones de corpora conversacionales (Briz, 1995 y Gallardo, 1998), las usadas en el corpus ACUAH (Análisis de la Conversación de la Universidad de Alcalá; Cestero, 2000a) o en las transcripciones publicadas del proyecto PRESEEA (Proyecto para el estudio sociolingüístico del español de España y América; Moreno, Cestero, Molina y Paredes, 2002). 
discurso verbal o durante discurso verbal, se pueden recoger entre doble paréntesis (( )) y colocar en el momento de su producción, salvo en los casos de sonido emitido a la vez que el discurso, en los que se indicará su extensión (inicio-fin de discurso con risa, bostezo, etc.).

C. Elementos cuasi-léxicos. Estos signos suelen ponerse entre paréntesis ( ) en el lugar en el que se producen. Se transcriben atendiendo a su producción, distinguiendo entre: signos vocálicos o vocalizaciones, signos consonánticos o consonantizaciones y signos combinados (vocalizaciones con consonantizaciones o consonantizaciones con vocalizaciones, dependiendo del sonido más tónico o alargado). Los signos consonánticos, atendiendo a su modo de articulación, pueden ser, básicamente, orales ( $T z$ de inicio de turno) o nasales ( $m m M$ de agrado) $\mathrm{y}$, según el punto de articulación, podemos clasificarlos como guturales $(\mathrm{OK}, \mathrm{OJ}$ de desagrado), palatales (Chss para pedir silencio), alveolares (TS de negación), dentales ( $T z$ de negación o inicio de turno), labiales ( $B r r$ de impaciencia) y nariales ( $N h$ de desacuerdo), simples o combinados.

Se suele transcribir con mayúscula el sonido más tónico o intenso y, en caso de producirse alargamiento de sonidos, éste se indica mediante su repetición.

D. Pausas y silencios. La ausencia de sonido suele indicarse entre paréntesis ( ), anotando las décimas o los segundos de duración. Es habitual distinguir entre:

* (-) Pausa (hasta 1 segundo, aproximadamente)

* (1,3)S Silencio (de 1 a 2 segundos, aproximadamente)

* (3)L Lapso (suspensión de sonido superior a 2 segundos)

Con respecto a las convenciones para recoger signos quinésicos, parece que el sistema empleado por los analistas de la conversación es el más efectivo ${ }^{32}$. Consiste en indicar el gesto - con una descripción sencilla y breve ((mirada fija en A)), ((frunciendo el entrecejo)), ((con leve sonrisa)), ((levantando la mano y señalando con el dedo a $B)$ ) — entre doble paréntesis. No obstante, dado que habitualmente utilizamos el doble paréntesis para determinados signos paralingüísticos, podría sustituirse esta convención, como hace Poyatos, por líneas oblicuas /mirada fija en A/, /frunciendo el entrecejo/. En el tipo de estudios que nos ocupa, parecen relevantes los siguientes tipos de signos quinésicos:

Marcadores quinésicos
A. Gestos
* gestos faciales, con especial atención a la sonrisa y a la mirada, y
* gestos corporales
B. Posturas y variaciones posturales.

A la hora de identificar, describir e inventariar los signos no verbales quinésicos, habitualmente se tiene en cuenta las distintas fases de su producción, a saber, la inicial o de formación, la central o de constitución y la final; no obstante, en el tipo de estudios que ahora trato, para simplificar, podría utilizarse sólo la final, que viene dada por la posición o el movimiento simple o continuo que define at signo y le confiere su valor significativo. Hemos de seleccionar un vocabulario específico para aludir a los diferentes movimientos y posiciones posibles y utilizarlo, de forma sistemática, en las descripciones: /mirada fija/, 
/movimiento giratorio hacia adelante/, /mirada hacia abajo transversal a izquierda/, /mueca con la boca hacia la derecha/, /guiño prolongado/, etc.; ello nos permitirá la identificación rápida del gesto.

Los indicadores proxémicos ${ }^{33}$, en forma, normalmente, de variaciones proxémicas significativas, se pueden poner entre llaves \{\} : \{acercamiento con el cuerpo al interlocutor\}, \{toque con la mano en el hombro del interlocutor\}, \{reorganización postural\}, etc.

Se anotará, por último, la incidencia de signos cronémicos cuando se considere preciso: duración de la mirada, prolongación de un gesto, etc.

Lo más apropiado para recoger en transcripción signos no verbales es situarlos en una línea por encima de los signos verbales. De esta manera, se puede marcar exactamente el lugar de su producción y su extensión, y queda bien reflejada la coestructuración de los signos de los diferentes sistemas. En caso de producirse simultáneamente signos lingüísticos, paralingüísticos y quinésicos, podemos suplementar las líneas, poniendo hasta tres: en la primera, los signos paralingüísticos que puedan incidir en la producción de otros signos paralingüísticos (cualidades del sonido, tipos de voz y esquema entonativo), en la segunda, el resto de signos paralingüísticos y los signos quinésicos y, en la tercera, el enunciado verbal.

3) Análisis del material

En este tipo de investigación, parece conveniente la realización de análisis cualitativos y cuantitativos de los datos.

El examen cualitativo de los enunciados irónicos nos permitirá identificar los indicadores que se utilizan para ironizar e interpretar la ironía, describir su forma y explicar su uso y función.

El análisis cuantitativo nos facilitará la realización de tipologías y clasificaciones, nos ayudará a comprobar la combinación de marcadores de distinto tipo (por ejemplo, cómo se combinan indicadores lingüísticos y paralingüísticos o lingüísticos y gestuales) mediante la aplicación de análisis factoriales o análisis de regresión múltiple y nos proporcionará frecuencias de aparición que nos permitirán generalizar o no.

Los dos tipos de análisis serán complementarios para que podamos conocer la incidencia fundamental o secundaria de los indicadores de ironización y su forma de coestructuración en la marcación de los enunciados irónicos.

4) Presentación de los resultados

Los resultados de un estudio sobre fenómenos como la ironía deben aparecer, a mi modo de ver, en forma de mecanismos de producción y de interpretación. Tales mecanismos son fácilmente convertibles en tácticas, en este caso de ironización, que permiten una aplicación inmediata a la enseñanza de español como lengua extranjera.

\section{A modo de conclusión}

En las páginas anteriores he intentado presentar, de forma resumida, qué es la comunicación no verbal y cuáles son los signos y sistemas que la integran y he tratado de explicar su incidencia en el proceso de comunicación humana. Mi objetivo no ha sido otro que mostrar la importancia que tienen los marcadores no verbales en la construcción e interpretación 
de enunciados y señalar los procedimientos básicos que han de guiar su estudio. Me sentiré totalmente satisfecha si las ideas y propuestas que acabo de ofrecer sirven de motivación o de ayuda para su investigación en relación con fenómenos pragmático-discursivos como la ironía verbal u otros de similares características.

\section{Referencias bibliográficas}

Atkinson, J. M. y J. Heritage (eds.) (1984): Structures of Social Action. Studies in Conversation Analysis. Cambridge, Cambridge University Press.

Briz Gómez, A. (coord.) (1995): La conversación coloquial (Materiales para su estudio). Valencia, Universitat de València. (Anejo XVI de la Revista Cuadernos de Filología).

Bruneau, T. J. (1980): "Cronemics and the verbal-nonverbal interface". En Key, M. (ed.) (1980): The Relationship of Verbal and Nonverbal Communication. The Hague, Mouton.

Cestero, A. M. (1996): "Funciones de la risa en la conversación en lengua española", Lingüistica Española Actual, XVIII, 2, págs. 279-289.

Cestero, A. M. (1999a): Comunicación no verbal y enseñanza de lenguas extranjeras. Madrid, Arco/ Libros.

Cestero, A. M. (1999b): Repertorio básico de signos no verbales del español. Madrid, Arco/Libros.

Cestero, A. M. (2000a): El intercambio de turnos de habla en la conversación. Alcalá de Henares, Servicio de Publicaciones de la Universidad de Alcalá.

Cestero, A. M. (2000b): "Comunicación no verbal y desarrollo de la expresión oral en la enseñanza y el aprendizaje de lenguas extranjeras", Carabela, 47, págs. 69-86.

Cestero, A. M. (2004): "La comunicación no verbal y el aprendizaje de lenguas extranjeras". En Sánchez Lobato, J. e I. Santos Gargallo (dirs.): Vademecum para la formación de profesores. Enseñar español como segunda lenguallengua extranjera. Madrid, SGEL, págs. 593-616.

Cestero, A. M. y M. Gil (1995a): "Comunicación no verbal: algunas diferencias culturales en la concepción, la estructuración y el uso del tiempo en India y en España, I", Cuadernos Cervantes, 4, págs. $49-53$.

Cestero, A. M. y M. Gil (1995b): "Comunicación no verbal: algunas diferencias culturales en la concepción, la estructuración y el uso del tiempo en India y en España, II", Cuadernos Cervantes, 5, págs. 62-66.

Gallardo Paúls, B. (1998): Comentario de textos conversacionales II. Los textos. Madrid, Arco/Libros.

Grice, H. (1975): "Logic and conversation". En Cole, P. y J. Morgan (eds.): Syntax and Semantics, vol. 3: Speech Acts. New York, Academic Press, págs. 41-58.

Grice. H. (1978): "Further notes on logic and conversation". En Cole, P. (ed.): Syntax and Semantics, vol. 9: Pragmatics. San Diego, Academic Press, págs. 113-127.

Hall, E. T. (1959): The Silent Language. Garden City, Doubleday and Company.

Hall, E. T. (1963): "A system for the notation of proxemic behavior", American Anthropologist, 65, 5, págs. 1003-1026.

Hall, E.T. (1966): The Hidden Dimension. Garden City, Doubleday and Company.

Hurley, D. S. (1992): "Issues in teaching Pragmatics, Prosody, and Non-Verbal Communication", Applied Linguistics, 13, 3, págs. 259-281.

Instituto Cervantes (1994): Plan curricular del Instituto Cervantes para la enseñanza del español como lengua extranjera. Alcalá de Henares, Instituto Cervantes.

Korte, B. (1997): Body Language in Literature. Toronto, University of Toronto Press.

Melrose, R. (1992): "Dialogue as process: Making communication 'real' in the second language classroom", International Review of Applied Linguistics in Language Teaching, 30, 3, págs. 
Moreno, F., Cestero, A. M., Molina, I. y F. Paredes (2002): La lengua hablada en Alcalá de Henares. Corpus PRESEEA-ALCALA I: Hablantes de instrucción superior. Alcalá de Henares, Servicio de Publicaciones de la Universidad de Alcalá. Edición en CD’Rom.

Navarro Tomás, T. (1985, 22 edición): Manual de pronunciación española. Madrid, CSIC.

Poyatos, F. (1972): "The communication system of the speaker-actor and his culture: A preliminary investigation", Linguistics, 83, págs. 64-86.

Poyatos, F. (1975): "Cultura, comunicación e interacción: hacia el contexto total del lenguaje y el hombre hispánicos, III", Yelmo, 22, págs. 14-16.

Poyatos, F. (1976): Man Beyond Words: Theory and Methodology of Nonverbal Communication. New York, New York State English Council, (NYSEC Monographs, 15).

Poyatos, F. (1992): "Nonverbal communication in foreign-language teaching: Theoretical and methodological perspectives". En Helbo, A. (ed.) (1992): Evaluation and Language Teaching: Essays in Honor of Frans van Passel. New York, Peter Lang, págs. 115-143.

Poyatos, F. (1993): Paralanguage: A Linguistic and Interdisciplinary Approach to Interactive Speech and Sounds. Amsterdam/Philadelphia, John Benjamins.

Poyatos, F. (1994a): La comunicación no verbal. Cultura, lenguaje y conversación. Madrid, Istmo.

Poyatos, F. (1994b): La comunicación no verbal. Paralenguaje, kinésica e interacción. Madrid, Istmo.

Poyatos, F. (1994c): "Paralenguaje y sonidos extrasomáticos en la novela: perspectivas semiótico-comunicativas a través de los estudios de comunicación no verbal". En Romera, J., Illera, A. y M. Garcia (eds.) (1994): Semiótica(s): Homenaje a Greimas. Madrid, Visor, págs. 141-155.

Poyatos, F. (1997): "El acto de lectura: su realidad verbal-no verbal", IRIA FLAVIA, XI.

Poyatos, F. (1998): "El paralenguaje en el Quijote: inventario completo y bases para su estudio", Signa, 7, págs. 293-318.

Poyatos, F. (1999): "Inventario kinésico del Quijote: corpus completo y bases para su estudio", Signa, 8 , págs. $281-326$.

Poyatos, F. (2006): "La enseñanza del español a extranjeros a través de los estudios de comunicación no verbal". En Cestero, A. M. (ed.): Lingüistica aplicada a la enseñanza de español como lengua extranjera: desarrollos recientes. Alcalá de Henares, Servicio de Publicaciones de la Universidad de Alcalá, págs. 27-46.

Quilis, A. (1993): Tratado de Fonología y Fonética españolas. Madrid, Gredos.

Reyes, G. (2002): Metapragmática. Valladolid, Servicio de Publicaciones de la Universidad de Valladolid.

Romera, J. (1994): "Repertorios extraverbales en la comunicación literaria”, Signa, 3, págs. 175-208.

Ruiz Gurillo, L., Marimón, C., Padilla, X. A. y L. Timofeeva (2004): "El Proyecto GRIALE para la ironía en español: conceptos previos", ELUA Estudios de Lingüistica Universidad de Alicante, 18, págs. 231-242.

Serrano, S. (1981): La semiótica. Una introducción a la teoria de los signos. Barcelona, Montesinos.

Sperber, D. y D. Wilson (1978): "Les ironies comme mentions", Póetique, 36, págs. 399-412.

Sperber, D. y D. Wilson (1981): "Irony and the use-mention distinction". En Cole, P. (ed.): Radical Pragmatics. New York, Academic Press, págs. 295-317.

Torres, M. Á. (1999): Aproximación pragmática a la ironía verbal. Cádiz, Servicio de Publicaciones de la Universidad de Cádiz.

Watson, O. M. (1970): Proxemic Behavior: A Cross Cultural Study. The Hague, Mouton. 